\title{
Title: Modeling and Designing Functionally Graded Ma- terial Components for Fabrication with Local Composition Control
}

\section{Authors:}

\begin{tabular}{|c|c|c|}
\hline \multicolumn{3}{|l|}{ T. R. Jackson } \\
\hline & Postal address: & MIT Room 5-430 \\
\hline & & Department of Ocean Engineering \\
\hline & & Cambridge MA 02139-4307, USA \\
\hline & Telephone number: & $617-253-7799$ \\
\hline & Fax number: & $617-253-8125$ \\
\hline & E-mail address: & trjackso@mit.edu \\
\hline \multicolumn{3}{|l|}{ H. Liu } \\
\hline & Postal address: & MIT Room 5-435 \\
\hline & & Department of Ocean Engineering \\
\hline & & Cambridge MA 02139-4307, USA \\
\hline & Telephone number: & $617-253-7798$ \\
\hline & Fax number: & $617-253-8125$ \\
\hline & E-mail address: & hliu@mit.edu \\
\hline \multicolumn{3}{|l|}{ N. M. Patrikalakis* } \\
\hline & Postal address: & MIT Room 5-428 \\
\hline & & Department of Ocean Engineering \\
\hline & & Cambridge MA 02139-4307, USA \\
\hline & Telephone number: & $617-253-4555$ \\
\hline & Fax number: & $617-253-8125$ \\
\hline & E-mail address: & nmp@mit.edu \\
\hline \multicolumn{3}{|l|}{ E. M. Sachs } \\
\hline & Postal address: & MIT Room 35-136 \\
\hline & & Department of Mechanical Engineering \\
\hline & & Cambridge MA 02139-4307, USA \\
\hline & Telephone number: & $617-253-5381$ \\
\hline & Fax number: & $617-253-0209$ \\
\hline & E-mail address: & sachs@mit.edu \\
\hline \multicolumn{3}{|l|}{ M. J. Cima } \\
\hline & Postal address: & MIT Room 12-011 \\
\hline & & Department of Materials Science and Engineering \\
\hline & Tolenhono number. & Cambridge MA 02139-4307, USA \\
\hline & & $617-253-6877$ \\
\hline & & $\begin{array}{l}\text { 617-258-6936 } \\
\text { mjcima@mit.edu }\end{array}$ \\
\hline
\end{tabular}

Keywords: Functional modeling, solid modeling, rapid prototyping. 


\title{
Modeling and Designing Functionally Graded Material Components for Fabrication with Local Composition Control
}

\author{
T. R. Jackson*, H. Liu*, N. M. Patrikalakis*, E. M. Sachs**, and M. J. Cima** \\ Massachusetts Institute of Technology \\ * Department of Ocean Engineering, Design Laboratory \\ ** Department of Mechanical Engineering, 3D Printing Laboratory \\ *** Department of Materials Science and Engineering \\ Cambridge, MA 02139-4307, USA \\ Design Laboratory Memorandum 98-11 \\ Issued: January 15, 1999
}
Copyright (c) 1998, Massachusetts Institute of Technology
All rights reserved

Keywords: Functional modeling, solid modeling, rapid prototyping.

\begin{abstract}
Solid Freeform Fabrication (SFF) processes have demonstrated the ability to produce parts with locally controlled composition. In the limit, processes such as $3 D$ Printing can create parts with composition control on a length scale of $100 \mu \mathrm{m}$. To exploit this potential, new methods to model, exchange, and process parts with local composition control need to be developed. An approach to modeling a part's geometry, topology, and composition is presented. This approach is based on subdividing the solid model into sub-regions and associating analytic composition blending functions with each region. These blending functions define the composition throughout the model as mixtures of the primary materials available to the SFF machine. Design tools based upon distance functions are also introduced, such as the specification of composition as a function of the distance from the surface of a part. Finally, the role of design rules restricting maximum and minimum concentrations is discussed.
\end{abstract}

\section{Introduction}

\section{Motivation}

With recent advances in Solid Freeform Fabrication (SFF), the ability to fabricate parts with Local Composition Control (LCC) is becoming a reality, opening the door to creating a whole new class of parts with graded compositions. Despite the advanced capabilities of these SFF machines, access to this new technology is limited by how information is represented, exchanged, and processed. Designers need new CAD tools to capture their ideas as models with graded compositions and manufacturers need algorithms capable of converting these models into machine instructions for their fabrication. To address these shortcomings, this project is developing new methods for the 
design, representation, exchange, and processing of models with graded composition. It is part of a larger project funded by the National Science Foundation titled "The Distributed Design and Fabrication of Metal Parts and Tools by 3D Printing. " The overall goal of this project is to identify the barriers preventing access to the unique capabilities of SFF processes and provide the requisite solutions. One of the identified barriers is this inability for designers and manufacturers to work with models of graded compositions. To address this issue, we have been researching methods to represent, design, exchange, and process these models with the goal of promoting the use and research of Local Composition Control by a wider audience of designers and researchers. ${ }^{1,2}$

\section{Solid Freeform Fabrication and Local Composition Control through 3D Printing}

Solid Freeform Fabrication (SFF) refers to a class of manufacturing processes that build objects in an additive fashion directly from a computer model. While some SFF processes are restricted to building in a single material at a time, most can be adapted to exercise some degree of control over the local composition. ${ }^{3-5}$ Among the most flexible is 3D Printing ${ }^{5}$ which can exercise control over composition on a near point-wise fashion through the selective placement of primitives of the various materials available to the machine. Such Local Composition Control (LCC) provides the opportunity to design and create parts with graded composition tailored for specific applications. Such compositions have become known as Functionally Graded Material (FGM) ${ }^{6,7}$ This work uses 3D Printing as a prototype SFF process to focus the development of an approach and tools which will hopefully be useful to the practitioners of other SFF processes.

Conventional 3D Printing manufactures a part by selectively binding powder together according to a computer model. The build cycle begins by spreading a layer of powder over the print bed. A print head then traverses the bed, selectively depositing binder over the regions corresponding to the interior of a slice of the computer model. After the layer is printed, the print bed is lowered and another layer of powder is spread. The process of spreading powder, depositing binder, and lowering the print bed is repeated, as shown in Figure 1(a), until the entire volume of the object is printed. At the end of the process, the bound powder becomes the manufactured object, effectively rendering the computer model as a physical object. Currently, metal and ceramic parts are being manufactured through 3D Printing, but the potential exists to build with any material supplied in powder form. A part manufactured with a single material is pictured in Figure 1(b).

Similar to how an ink-jet printer prints color documents, 3D Printing can achieve LCC with multiple materials. This is accomplished by using a print-head with several jets, as shown in Figure 2, each depositing binders and/or slurries of unique material. By varying the pattern in which the jets deposit material on the powder-bed, the material composition can be controlled on the scale of the binder droplets $(100 \mu m)$. Regions of uniform and graded compositions can be created in a manner analogous to how continuous tone images are rendered on a hard-copy device from primary colors. With this capability, graded compositions can be designed along with the geometry of the part, tailoring the part's physical properties for a specific purpose or function.

The capability of LCC and fabrication of FGM parts could be utilized by a wide variety of industries. Applications could range from multi-color visualization models to functional parts and tools. For example, the potential to convey additional tissue information through multi-color medical models would increase their usefulness in medical applications such as surgical planning. ${ }^{8}$ Capable of producing functional parts, graded compositions could also be useful in controlling the mechanical properties of parts and tools at a local scale, potentially reducing distortion due to internal stresses, increasing hardness at points of greatest wear, or resisting failure, for example by locally controlled toughening. ${ }^{7}$ The application of FGM compositions to drug delivery devices is even being studied as a means to achieve optimal, controlled release of medicine into a patient. ${ }^{9}$ 


\section{Traditional Solid Modeling and Information Flow for SFF}

Traditionally, systems used for 3D object representation are based on one of three different classes of solid modeling methods: Decomposition Models, Constructive Solid Geometry (CSG), or Boundary Representation (B-rep). ${ }^{10}$ Each has its merits, but their current implementations in the CAD/CAM industry do not easily permit manufacturing parts with LCC.

The first class of methods, Decomposition Models, model objects by subdividing space into multiple sub-regions. These methods are most commonly used for associating physical properties with sub-regions for the purposes of analysis and visualization, such as in finite element analysis, medical data rendering, and geophysical or oceanographic surveys.

The latter two classes of methods, CSG and B-rep, are more commonly used in design systems. The CSG approach represents a solid object by applying Boolean operations on primitive geometric shapes such as rectangular boxes, cylinders, spheres and tori, ${ }^{11,12}$ allowing a designer to define a model from a collection of simple primitives. For complicated curved models, however, the most prevalent representation in the CAD industry is B-rep due to its flexibility to precisely describe complex geometric models in a concise manner. ${ }^{13}$ In a B-rep model, the boundary of a region is defined by a shell of faces which each are bounded by loops of edges. To define the geometry of the B-rep models, non-uniform rational B-spline (NURBS) formulations are generally chosen for their generality, flexibility, and their inclusion into neutral data exchange standards (such as IGES ${ }^{14}$ and STEP ${ }^{15}$ ). The B-rep method is convenient for supporting important geometric operations useful for design, such as visualization, splitting, joining, subdividing, and Boolean operations with solids. ${ }^{16,17}$

In current SFF practice, a designer creates a model on a system employing one of the above methods. Next, the boundary of the model is tessellated into facets, usually as a collection of triangles in the format known as an .STL file. ${ }^{18}$ This faceted boundary representation of the model is then processed into machine instructions to drive the fabrication process. Only conveying information about the facets bounding the internal region of the model, material information about the model is not explicitly represented.

With the possibility of fabricating graded compositions through LCC, methods to represent, design, and process models need to be reconsidered and extended. Although capable of conveying physical properties, traditional decomposition models are not as convenient or accurate as the CSG or B-rep methods for representing and designing the geometry of a model. In addition, decomposition methods such as voxel models are defined relative to a fixed coordinate system making transformations (such as rotations) cumbersome and inaccurate. On the other hand, CSG and B-rep models permit modeling of uniform compositions within regions allowing representation of composite structures. To represent a region of graded composition within an existing system, multiple shells, each of uniform composition, would have to be assembled by a designer to approximate the desired grading. Besides design issues, the manner in which models are exchanged with the fabrication process needs to be adapted to not only convey material information, but also the grading of the material over space if the design and fabrication of FGM parts through LCC is to be achieved.

These obstacles have been identified by various research groups. In May 1997, Kumar and Dutta ${ }^{19}$ proposed modeling multi-material objects as generalized B-rep models by using r-sets extended to include composition $\left(r_{m}\right.$ sets) with accompanying Boolean operators to build models. Although initially presented as a method for representing regions of uniform composition, they conclude that this approach can be extended to handle continuous (or graded) compositions. In January 1998, Sachs et $a l^{1}$ outlined our approach to model FGM models using the cell tuple data structure with volumetric, FGM cells. Later in August 1998 at the SFF Symposium, Pegna and Safi ${ }^{20}$ proposed taking advantage of the existing tools for handling volumetric data sets (common in medical imaging and finite element analysis) by representing multi-material models as point sets (including Cartesian coordinates plus material composition). Through such an approach, objects could be represented as decomposition models and readily represented and visualized through existing software. Decomposition modeling, however, is cumbersome during design because it 
does not maintain topological information about the model and is not as memory efficient as the more commonly used B-rep methods which incorporate parametric representations for free-form curves and surfaces (NURBS). At the same SFF Symposium, Jackson et a ${ }^{2}$ expanded upon the paper by Sachs et $a l^{1}$ and presented our cell tuple approach to modeling FGM parts in more detail along with methods to design FGM parts as a function of distance from the part's surface or a specific feature. Later in December 1998, Kumar and Dutta ${ }^{21}$ reported extensions to their modeling approach to handle heterogeneous objects with graded composition following a power law. The work presented here expands upon the paper by Jackson $e l a l^{2}$ presented at the SFF Symposium.

\section{Method}

\section{Representation of FGM Models}

In order to represent an object within the computer, a data structure representing all of the relevant information for its fabrication must be established. In state-of-the-art CAD systems, solid modeling methods maintain information about an object's geometry (shape) and topology (adjacency relationships between the geometric elements of its surface). ${ }^{10}$ Some CAD systems also provide the capability to associate material information with regions, facilitating the representation of composite structures. With the possibility of fabrication with LCC, a solid modeling method for 3D Printing must go one step further and represent smoothly varying compositions. Similar to how sculpted geometry can be represented as analytic functions (such as NURBS surfaces) methods to analytically describe how an FGM composition varies within a part need to be established. To provide this capability, an FGM solid modeling method must decompose the interior of the object into simpler sub-regions, each of which references information about the composition variation over its domain. To accomplish this goal, an FGM solid modeling method based on a representation known as the cell-tuple data structure has been developed in a prototype form. ${ }^{22}$ This structure naturally lends itself to the representation of models in terms of sub-regions over which the FGM information can be incorporated, similar to how the geometric information is maintained.

In the traditional cell-tuple structure, a model $M$ is decomposed into a set of cells $C$ with each cell $c_{\kappa}$ representing a topological feature in the model, such as a vertex, edge, face or region. The topology of the model (or how the cells are connected together) is maintained by a graph of cells $T$. The shape, position, and orientation of the model are determined by geometric information associated with each cell. Figures 3(a)-(c) illustrate this concept for a simple model of a tetrahedron with cells representing the different topological elements whose incidence relationships are maintained through a graph of cells. For larger models, the interior may be decomposed into a single region, as in a traditional B-rep model or an .STL file, or subdivided into many smaller regions, similar to a finite element mesh. Each point $\mathbf{x}$ in the model lies within exactly one cell.

To represent a FGM model within the cell-tuple structure, composition information as well as geometric information is associated with each cell. This information begins with the concept of a material space $\mathbf{M}$ spanning the $d_{m}$ primary materials available to an SFF machine capable of LCC. The composition of the model is represented as a vector valued function $\mathbf{m}(\mathbf{x})$ defined over the model's interior. Each component $m_{j}$ of $\mathbf{m}$ represents the volume fraction of the corresponding material in the material system present at point $\mathbf{x}$ within the model.

There are many possible approaches to defining the composition function $\mathbf{m}(\mathbf{x})$. For parts similar to traditional composite structures, constant values can be associated with each cell $\kappa$ within the model: $\mathbf{m}(\mathbf{x})=\mathbf{m}_{\kappa}$ for $\mathbf{x} \subset c_{\kappa}$. For graded compositions, however, analytic functions must be defined, capable of representing smooth variations in the volume fractions of the materials over the domain of each sub-region.

With the cell-tuple structure's capability to represent models as subdivided manifolds, models can be arbitrarily subdivided into topologically simpler domains over which shape and composition functions can be more readily defined analytically. In our approach, we are simplifying the problem 
by beginning with models subdivided into tetrahedral meshes, permitting the use of standard meshing algorithms to convert traditional solid models to our cell-tuple representation. However, in the general formulation below the faces of these tetrahedral meshes may be curved. (In all examples of this paper we nevertheless use planar faces for all such elements). Over each cell's domain $c_{\kappa}$, the shape and composition is formulated in terms of a set of control points $\left\{\mathbf{x}_{\kappa, \mathbf{i}}|| \mathbf{i} \mid=n_{g}\right\}$ and control compositions $\left\{\mathbf{m}_{\kappa, \mathbf{i}}|\mathbf{i}|=n_{m}\right\}$ which are blended with the barycentric Bernstein polynomials: ${ }^{23}$

$$
\left[\mathbf{x}_{\kappa}(\mathbf{u}), \mathbf{m}_{\kappa}(\mathbf{u})\right]=\left[\sum_{|\mathbf{i}|=n_{g}} B_{\mathbf{i}}^{n_{g}}(\mathbf{u}) \mathbf{x}_{\kappa, \mathbf{i}}, \sum_{|\mathbf{i}|=n_{m}} B_{\mathbf{i}}^{n_{m}}(\mathbf{u}) \mathbf{m}_{\kappa, \mathbf{i}}\right]
$$

where $n_{g}$, and $n_{m}$ are the degrees of variation in shape and composition, $|\mathbf{i}|=i_{0}+i_{1}+\cdots+i_{k}$ and $k$ is the dimension of cell $\kappa$.

$B_{\mathbf{i}}^{n}(\mathbf{u})$ represents the $\mathbf{i}^{\text {th }}$ Bernstein polynomial ${ }^{23}$ of degree $n$ and each polynomial is defined over a parametric domain in terms of barycentric coordinates:

$$
B_{\mathbf{i}}^{n}(\mathbf{u})=\left[\frac{(n !)}{\left(i_{0} !\right)\left(i_{1} !\right) \cdots\left(i_{k} !\right)}\right]\left[\left(u_{0}^{i_{0}}\right)\left(u_{1}^{i_{1}}\right) \cdots\left(u_{k}^{i_{k}}\right)\right]
$$

$\mathbf{u}=\left[u_{0}, u_{1}, \ldots, u_{k}\right]$ represents the barycentric coordinates of a point in the domain and satisfies the condition $|\mathbf{u}|=u_{0}+u_{1}+\cdots+u_{k}=1$. For linear variation $(n=1)$ over a tetrahedral $(k=3)$ domain, the functions take the form:

$$
\left[\begin{array}{l}
B_{1000}^{1}\left(u_{0}, u_{1}, u_{2}, u_{3}\right) \\
B_{0100}^{1}\left(u_{0}, u_{1}, u_{2}, u_{3}\right) \\
B_{0010}^{1}\left(u_{0}, u_{1}, u_{2}, u_{3}\right) \\
B_{0001}^{1}\left(u_{0}, u_{1}, u_{2}, u_{3}\right)
\end{array}\right]=\left[\begin{array}{l}
u_{0} \\
u_{1} \\
u_{2} \\
u_{3}
\end{array}\right]
$$

An example of mapping for linear geometry and composition variation for a tetrahedral cell using the above blending functions is illustrated in Figures 4(a)-(e). Figure 4(a) shows the parametric domain U of the cell: a tetrahedron. The barycentric coordinates of a point are computed as the ratio of the volumes of the tetrahedra defined by a parametric point within its domain and the four vertices of the domain:

$\mathbf{u}=\frac{1}{\operatorname{Vol}\left(v_{0}, v_{1}, v_{2}, v_{3}\right)}\left[\operatorname{Vol}\left(v_{p}, v_{1}, v_{2}, v_{3}\right), \operatorname{Vol}\left(v_{0}, v_{p}, v_{2}, v_{3}\right), \operatorname{Vol}\left(v_{0}, v_{1}, v_{p}, v_{3}\right), \operatorname{Vol}\left(v_{0}, v_{1}, v_{2}, v_{p}\right)\right]$

The control points $\left\{\mathbf{x}_{\kappa, \mathbf{i}}|| \mathbf{i} \mid=n_{g}\right\}$ of the cell are defined in model space X, as given in Figure 4(b), resulting in linear geometry interpolating these points (Figure 4(c)). For this example, we assume a material system of $d_{m}=2$ materials. The control compositions $\left\{\mathbf{m}_{\kappa, \mathbf{i}}|| \mathbf{i} \mid=n_{m}\right\}$ defining the cell's composition are defined within the material space M, as shown in Figure 4(d). Materials $m_{1}$ and $m_{2}$ are represented by black and white, respectively. The resulting, linearly graded composition is illustrated over the cell's geometry in model space in Figure 4(e).

The above example demonstrates linear variations over a 3D cell, but the barycentric polynomials can be of higher degree as well as defined in other dimensions. For example, for quadratic variation $(n=2)$ over a triangular $(k=2)$ domain can be used to define the geometry of a curved face. In this case, the blending functions take the form of:

$$
\left[\begin{array}{c}
B_{200}^{2}\left(u_{0}, u_{1}, u_{2}\right) \\
B_{110}^{2}\left(u_{0}, u_{1}, u_{2}\right) \\
B_{020}^{2}\left(u_{0}, u_{1}, u_{2}\right) \\
B_{011}^{2}\left(u_{0}, u_{1}, u_{2}\right) \\
B_{002}^{2}\left(u_{0}, u_{1}, u_{2}\right) \\
B_{101}^{2}\left(u_{0}, u_{1}, u_{2}\right)
\end{array}\right]=\left[\begin{array}{c}
u_{0}^{2} \\
2 u_{0} u_{1} \\
u_{1}^{2} \\
2 u_{1} u_{2} \\
u_{2}^{2} \\
2 u_{0} u_{1}
\end{array}\right]
$$

The higher degree requires additional control points or control compositions to be defined, but allows smooth variations to be represented. 
Conceptually, the composition $\mathbf{m}(\mathbf{u})$ at a point $\mathbf{x}=\mathbf{x}(\mathbf{u})$ can be considered as a blend of the control compositions with their influence determined by the value of their basis functions, analogous to the representation of NURBS surfaces with a mesh of control points. ${ }^{23}$ By defining compositions in terms of the Bernstein polynomials, the degree $n_{m}$ of composition variation is arbitrary, permitting the representation of regions of piece-wise uniform composition as well as higher order graded composition, as shown in Figure 5.

Although beginning with meshed tetrahedral models, the cell-tuple data structure is sufficiently general to represent a model as any valid subdivided manifold. This will permit FGM objects to be efficiently and accurately modeled from a suitable collection of FGM cells. Regions of uniform composition, for example, could be represented with a single region cell of constant composition, bounded by an arbitrary number of faces (similar to the traditional B-rep methods). For graded regions, a collection of FGM cells starting with the tetrahedron can be defined, permitting different subdivision schemes of the object's interior. Hexahedral, wedge, and pyramid finite elements, for example, could eventually be defined with formulations for their geometry and graded composition in terms of tensor product B-splines or mixed tensor product B-splines and barycentric polynomials. ${ }^{23}$ These formulations permit the specification of continuous compositions at the interface of the cells using elementary properties of Bernstein polynomials. However, specification of compositions with higher order derivative continuity is more complex and is not addressed in this paper.

The main components of our FGM solid modeling system are shown in Figure 6. Models created on a state-of-the-art CAD system are meshed into finite elements and then loaded into the data structure. As described above, the model's topology is maintained by the cell-tuple structure as a graph of cells. Each cell in the model references information about its own geometry and composition. The material system is composed of the primary materials available to the SFF machine. Through the FGM Modeler, the designer is able to specify the grading of the composition. The SFF machine is assumed to have the capability to selectively place primitives of each material during the build process. Similar to how an ink-jet printer strategically places drops of the primary colors on a page to represent continuous tones, the model processor will generate the machine instructions to accurately fabricate the desired compositions. The processing of FGM models for fabrication is a subject of current research and details on these algorithms will be included in a forthcoming paper. We anticipate capturing limitations in the manufacturing process as Design Rules which can be used to evaluate models during the design phase, before fabrication.

\section{Design of FGM Models}

With each control composition in our data structure representing a degree of freedom, the task of designing the FGM by assigning values to each can be non-intuitive and confusing at the very least, if not impossible considering that a model may have millions of FGM cells. To aid the designer in this task, tools for simplifying and automating the design of FGM compositions are being developed. One approach being explored is the design of compositions in terms of distance functions.

The design of FGM compositions in terms of distance functions begins with the selection of a feature by reference to which the composition will be designed. This may be a fixed reference in the model space, such as a point, line, or plane, or a feature of the model, such as a particular face or its entire boundary. Next, the designer specifies a variation for the FGM in terms of distance from the feature: $\mathbf{m}\left(x^{*}\right)=\mathbf{m}\left(r\left(\mathbf{x}^{*}\right)\right)$, where $r$ is the distance of a query point $\mathbf{x}^{*}$ from the reference feature. With a reference feature selected and a FGM variation designed, an algorithm automatically visits and assigns the control compositions for each cell, thereby defining the FGM throughout the model. 


\section{Efficiently designing FGM as a function of distance from the model's boundary}

Not only should tools for designing compositions be useful and relatively straight forward to use, they must also perform their tasks in an efficient manner. In our representation, a model with a complicated shape and many control compositions may require a prohibitively long time to define the FGM if implemented inefficiently. Consider the design of FGM as a function of distance from the model's boundary. The algorithm assigning the control compositions must compute the minimum distance from each query point (corresponding to a control composition) to the boundary of the model. With the potential of a model having a large number of query points in its interior, the search through all of the boundary facets for the closest facet for all these points may be prohibitively time consuming. To address this issue, an efficient algorithm to compute the minimum distance from a point to the boundary through bucket sorting is developed. ${ }^{24}$ Instead of performing an exhaustive search on all of the boundary facets, this bucketing technique allows us to limit the scope of the search for the facet nearest a query point by initially sorting the facets according to their positions.

The bucketing approach to computing the minimum distance begins by dividing the $3 \mathrm{D}$ domain of the model into equal-sized cubical subdomains (buckets). References from each bucket to boundary facets intersecting the bucket are then established. This sorting process occurs once during the design phase and requires computation time $T_{s}$ proportional to the number of boundary facets $n_{b}: T_{s}=O\left(n_{b}\right)$, where $O($ ) represents an asymptotic upper bound for the computation time. The search for the nearest facet begins with checking if the bucket containing the query point contains any boundary facets. If yes, the minimum distance to all facets contained in this bucket is computed and algorithm terminates. If not, a layer of buckets adjacent to the above bucket and with thickness one bucket is considered. Buckets in this layer are checked to see if they contain any boundary facets. If yes, the minimum distance to all boundary facets contained in this layer of buckets is computed and the algorithm terminates. If not, the algorithm continues by considering the next layer of buckets around the previous layer of buckets and continues as before. The time required to find this nearest facet $T_{c}$ is proportional to the number of buckets checked $n_{c}$ until a non-empty bucket is found and the number of facets $n_{l}$ in all of the buckets at that layer; $T_{c}=O\left(n_{c}+n_{l}\right)$.

Compared to the exhaustive search method, we observe a significant reduction in the time required to compute the minimum distance. For the exhaustive search approach to computing the minimum distances for $m_{q}$ query points, the distance between each query point and each facet needs to be checked and the time complexity $T_{d}$ is simply: $T_{d}=O\left(m_{q} \cdot n_{b}\right)$. By initially sorting the boundary facets, the bucketing approach yields a time complexity $T_{b d}$ of

$$
T_{b d}=T_{s}+m_{q} T_{c}=O\left(n_{b}+m_{q}\left(n_{c}+n_{l}\right)\right) .
$$

In the worst case for the bucketing method, the search for the nearest facet might degenerate to an exhaustive search of all of the boundary facets: $T_{b d}^{\text {worst }}=O\left(T_{d}\right)$. Typically, however, we observe that $n_{l} \ll n_{b}$ and $n_{c} \ll n_{b}$, yielding a significant improvement in running time: $T_{b d}^{\text {observed }} \ll T_{d}$. This trend in the observed running time is illustrated in Figure 7(a) in which the time required by an exhaustive search algorithm is compared to the bucketing algorithm for a number of query points distributed throughout the part shown in Figure 7(b).

\section{Design Rules}

The ability to represent complex FGMs does not guarantee the ability to manufacture the part. Due to the limited accuracy and resolution inherent in any process, some FGM models may not be fabricated to the designer's satisfaction. To avoid this situation, a set of "Design Rules" governing the design of FGMs need to be established. These Design Rules will be based upon the limitations in the manufacturing process (resolution, accuracy, layer thickness, etc.) and will be used to evaluate models for their manufacturability. If a CAD model violates the Design Rules, the designer will be informed of the violation and will have the opportunity to redesign the 
model without the costly and disappointing process of manufacturing an unacceptable part. By providing tools to enforce Design Rule checking, some of the burden of ensuring part quality is reduced, allowing the designer to work with FGMs without being an expert in the manufacturing capabilities of the machine. Similar concepts involving minimum feature size were explored for macro-texturing for 3D Printing. ${ }^{25}$ For FGM models, two classes of Design Rules are being explored, governing the model's composition and its rate of change. Only the former is described in this paper.

\section{Design Rules limiting maximum and minimum composition}

Depending upon the material system used to fabricate the part, there is a limit to the volume fraction of each material that can be present at a point and still guarantee successful fabrication. Hence, the first foreseen set of Design Rules would limit the maximum and minimum volume fraction of each material in a FGM model to within the fabrication limits of the process.

In 3D Printing, for example, the material system is composed of the powder in the print bed, several additional materials which are jetted into the powder bed from the print head, and voids (representing porosity). A set of Design Rules for 3D Printing would limit the volume fraction of each of these materials based upon the limitations of the process. The selection of the powder material, size of powder, and powder shape substantially determines the packing density of the powder bed created by spreading that powder. However, some small degree of control over the powder bed density may be exercised during the layering by changing the parameters of the layering process. To hold the powder together, a restriction is imposed on the minimum presence of binder to guarantee sufficient strength in the "green part" (the printed part before post processing steps). In addition, all jetted material (binding or otherwise) is delivered within a liquid vehicle (either as suspended particles or in solution), placing a maximum limit on the amount of each material which can be delivered. This limit is due in part to the maximum concentration of material which can be suspended or dissolved in the liquid as well as the maximum solids loading which can be successfully jetted through the printing nozzles. After printing, the liquid evaporates leaving behind deposited material. The volume not filled by material is left as voids, which are typically present in green components fabricated through 3D Printing. During the design, the designer has the freedom of specifying the amount of solid binder or other material within the limits of these Design Rules.

Enforcement of the Design Rules involves restricting the assignment of the FGM such that $D R_{j, \min } \leq m_{j}(\mathbf{x}) \leq D R_{j, \max }$, where $j$ is the material index and $m_{j}(\mathbf{x})$ is the volume fraction of material $j$ at a point $\mathbf{x}$. For a system of four materials (the powder bed, two deposited materials, and voids), a hypothetical set of Design Rules governing the permissible compositions is defined and applied to the example FGM model in Figure 8. For this example, the volume fractions of materials $m_{0}$ (powder) and $m_{1}$ (binder) are uniform throughout the part and the volume fractions of materials $m_{2}$ (non-binding material) grades linearly from midplane to the top and bottom faces. The porosity in the part is represented by $m_{3}$, which is given by $m_{3}=1-m_{0}-m_{1}-m_{2}$. Materials $m_{0}$ and $m_{1}$ satisfy the Design Rules throughout the part. Material $m_{2}$, however, violates the maximum limit specified by Design Rule $D R_{2}$, perhaps imposed by the maximum amount of material $m_{2}$ which can be maintained in the liquid. Due to the limitations in the fabrication process, the specified FGM cannot be manufactured and must be redesigned. By capturing these limitations as rules, the FGM can be automatically checked during the design phase for compliance, restricting compositions to those which can be successfully fabricated and reducing the burden of understanding the LCC process on the part of the designer. 


\section{Results}

\section{Representation, Visualization, and Design of FGM Models}

A solid modeling system capable of representing models subdivided into topological cells is implemented. Although sufficiently general to handle arbitrary B-rep models, the current implementation allows the representation of faceted B-rep models, tetrahedral meshes, or a mixture of the two. Models generated on a traditional CAD system can be converted into a triangulated B-rep model (.STL format) or subdivided into tetrahedra using a meshing algorithm and then loaded into the FGM modeler. The geometry and composition of each cell are defined in terms of sets of control points and compositions, respectively, and blended with the barycentric Bernstein polynomials of the appropriate dimension and specified degree.

To represent the composition, a material system maintaining $d_{m}$ materials in the model is defined. Anticipating the use of a variety of material systems, the number of materials is arbitrary and will depend on the application and SFF process.

At the current stage of development, models can be viewed in terms as a collection of cells shaded according to the topological entity they represent, as their control points, as their control compositions, or as a collection of cells shaded according to the variation of their composition over their domain. Inspection of the interior is accomplished through sliced views and "shrunken cell" views of the model. In the shrunken cell visualization, the parametric domain over which each cell is rendered is restricted, resulting in an exploded view of the model decomposed into its collection of cells (see Figures 3(b), 9(d), and 10(d)). Color assignment for each material is arbitrary, allowing the distribution of each material to be displayed individually or in combinations with each other.

Finally, in order to capture the designer's composition intent, design methods based upon distance functions have been implemented. Designers can specify desired composition gradings as functions of distance from points, lines, planes, or the model's external boundary.

\section{Design Example 1: FGM Design of a Pulley.}

Our first example illustrates the design of a FGM pulley with increased hardness near the hub and rim, the surfaces most susceptible to wear. To fabricate this part, a designer could use a 3D Printer capable of selectively placing droplets of binder and carbide slurry into a stainless steel powder bed. Due to fact that the binder and carbide are delivered in a liquid vehicle, voids will be left behind as the liquid evaporates. This porosity must also be represented, occupying the remainder of the volume. During post processing steps, including sintering and/or infiltration, this porosity can be closed or filled.

The design process begins by defining the geometry of the part on a state-of-the-art solid modeler. The model is then meshed into tetrahedra and loaded into the FGM modeler (Figure 9(a)). Next, the designer defines the axis of rotation as the reference feature and designs a composition variation as a function of distance from this line, as shown in Figure 9(b). With this information, the degrees of freedom (control compositions) of the model are assigned, producing the desired FGM throughout the model, as illustrated in Figure 9(c). The decomposition of the model into FGM cells is shown in Figure 9(d).

\section{Design Example 2: FGM Design of a Drug Delivery Device.}

Our second example is the design of a drug delivery device with controlled release properties. Recognizing that the rate a drug is released from a pill is related to the rate at which it dissolves, the rate of drug release over time can be controlled by either tailoring the distribution of the drug within the pill or the permeability of the pill. Fabrication of such a device can be achieved through LCC. Wu et at demonstrated this capability with the controlled release from a device fabricated through 3D Printing by selectively depositing droplets of drug into a powder bed of the 
pill's matrix. As with the previous example, the binder and drug would be delivered in a liquid vehicle, resulting in a less than fully dense part (represented by voids) after the liquid evaporates.

As before, the design process begins by creating a solid model of the pill's geometry which is then meshed and loaded into the FGM modeler (Figure 10(a)). With the simplifying assumption that the time at which a point in the model is exposed for drug release is roughly proportional to its distance from the boundary, a graded profile for the drug distribution is specified as a function of distance from the pill's boundary (Figure 10(b)). Using the bucket sorting method previously described, the control compositions in the model are then assigned, defining the FGM over the drug delivery device. The concentration of drug within the FGM pill is illustrated over an exploded view of the model's cells Figure 10(c) and over the surfaces of the internal FGM tetrahedral cell in a sliced view of the pill in Figure 10(d).

\section{Discussion}

New developments in SFF processes promise the capability of fabricating parts with locally controlled composition. This will open the door to the possibility of fabricating a whole new class of parts consisting of graded compositions or FGM. To realize this potential, however, current $\mathrm{CAD} / \mathrm{CAM}$ methods need to be extended to enable designers and researchers to model, design, and exchange FGM parts. In our work, we are attempting to extend modeling techniques used for the geometric modeling of sculpted shapes to representing graded compositions.

Currently, a prototype solid modeling system based on the cell-tuple data structure has been implemented in $C++$, using the $O p e n G L^{26}$ graphics library for visualization. The cell tuple structure maintains the topological relationship between cells representing regions, faces, edges, and vertices in the model. The geometry and composition of each cell is defined in terms of sets of control points and control compositions which are blended through the barycentric Bernstein polynomials of the appropriate dimensions. The degrees of variation in geometry $n_{g}$ and composition $n_{m}$ can be chosen independently of each other. Although capable of representing curved geometry, only linear geometries have been presented in this paper since we are focusing on the representation and design of graded compositions. We use a state-of-the-art solid modeling system to define the geometry of the object and then convert it to our representation by meshing it into sub-regions using a state-of-the-art finite element meshing system.

For the example of the pill, a solid model of the pill was saved in a neutral B-rep format (IGES) and then meshed into finite elements. This meshing step took 17 seconds on a personal computer running at $266 \mathrm{MHz}$ with 128 megabytes of RAM. This conversion produced a file containing the vertices of the model and tetrahedral elements interpolating these vertices. Our modeler then loaded this file and derived the necessary topological data for the cell tuple structure. For the pill example, the process of reconstructing the topology for its 27809 cells of dimensions 0 to 3 (1329 vertices, 7813 edges, 12576 faces, and 6,091 regions) took 121 seconds on the same machine. The cell tuple graph maintaining the topological information contained 150912 nodes. Once the pill model was loaded, equations for the FGM variation from the boundary were defined and the control compositions within each cell automatically defined. For linear variation through each cell, the process of assigning compositions using the bucketing approach took 60 seconds, whereas the exhaustive search approach took 298 seconds.

Beginning with models derived from triangulated, STL models, we can expect FGM models with uniform meshes to be large. For simplicity of analysis, consider a cube subdivided into a structured mesh of tetrahedra. The relationship between the number of tetrahedra $n_{\text {tet }}$ in the mesh and the number of boundary facets $n_{b}$ in the STL representation is: $n_{\text {tet }}=5\left(n_{b} / 12\right)^{3 / 2}$. For a small STL model of only 9408 facets, the corresponding FGM model of the cube with a uniform mesh would have 497225 cells of dimensions 0 to 3 (24389 vertices, 138852 edges, 224224 faces, 109760 regions) and require a graph with 2690688 nodes to maintain the topology. Although refinements in implementation may improve performance, the data required to maintain the topological, geometric, and material information will nevertheless become prohibitively large if we naively apply meshing 
routines to convert STL or B-rep models to our FGM representation, especially if we assume $n_{\text {tet }} \propto n_{b}^{3 / 2}$ to be a reasonable estimate for the growth of more general STL models. Decisions about how a model should be subdivided (how and where to place the cells capable of representing graded compositions) should reflect the desired variation in FGM as well as the model's geometry. A region of uniform composition, for instance, is efficiently represented as a single region cell of uniform composition bounded by an arbitrary number of faces. Blending FGM cells should only be applied where the representation is needed for smoothly varying composition. These concepts are analogous to state-of-the-art solid modeling in which planar facets are used for flat faces and higher order, parametric surfaces are used for curved faces. By subdividing models with some consideration to its geometry and desired composition variation, and with the development of a larger library of FGM cells, the size of FGM models within our framework should became manageable. Methods to efficiently subdivide models into optimal subregions, however, remains an open issue.

Through our approach, we are attempting to maintain the flexibility in geometric design that a traditional B-rep modeler affords while allowing models to be arbitrarily decomposed into subregions of graded compositions to efficiently and accurately model FGM parts. In addition, we anticipate the properties which make the Bernstein polynomials so appealing to geometric design (such as the convex hull and subdivision properties ${ }^{23}$ ) will be equally useful in the design and interrogation of FGM compositions. Although initially working with triangulated/tetrahedral subdomains, the lessons learned from modeling FGM with Bernstein polynomials will be applicable to more general models containing FGM cells based upon the more general NURBS and mixed NURBS-barycentric formulations.

There are many possible directions for future work in the area of FGM modeling. These include implementing a larger library of cells (hexahedral cells, for example), proposing new design and visualization methods, and establishing efficient and logical methods for subdividing traditional B-rep models into sub-regions of graded composition. Methods to adaptively subdivide cells into smaller cells during FGM design, for instance, could reduce the model size to the minimum number of cells necessary to accurately approximate the exact distance functions. For the design of the FGM models, higher level and more abstract functional specification of the composition is an important topic of further research. For example, the designer might wish to specify the FGM directly in terms of trade offs between hardness and toughness and have the compositions automatically derived from this information by the design system. As FGM model processing algorithms are implemented for fabricating FGM parts through 3D Printing, LCC material systems will need to be established and their limitations captured as Design Rules and applied to FGM models. Finally, memory compression issues in our data structures for representing and processing FGM should be explored to handle large, complex models.

In conclusion, we have been addressing the obstacles to accessing LCC fabrication at MIT. These obstacles can be divided into two general areas: (1) the representation and design of ideal, continuous FGM models and (2) the accurate processing of these models into machine instructions for fabrication through LCC. At the current stage of our project, a prototype modeling system exists capable of accepting a triangulated 3D model from an existing CAD system, representing it as a FGM solid model, and permitting the design of its graded composition variation according to distance functions. While exploring issues in modeling FGM parts, refining the implementation of the data structure, and extending the modeler's functionality, our future work will also include addressing issues of processing these models into machine instructions to drive the MIT 3D Printer, enabling the automatic fabrication of FGM parts. Through this work, our goal to provide a pathway for designers to design and model FGM components and then see them fabricated through LCC, enabling a promising new technology for the creation of an entirely new class of parts. 


\section{Acknowledgements}

Support of this project was provided in part by the National Science Foundation (grant \#DMI9617750) and by the Office of Naval Research (grant \#N00014-96-1-0857). CAD models for this paper were generated with SolidWorks ${ }^{T M}$, meshed with Algor $^{T M}$, and then loaded into our special purpose FGM modeler.

\section{References}

[1] E. M. Sachs, N. M. Patrikalakis, D. Boning, M. J. Cima, T. R. Jackson, and R. Resnick. The distributed design and fabrication of metal parts and tooling by 3D Printing. In Proceedings of the 1998 NSF Design and Manufacturing Grantees Conference, Cintermex Conference Center, Monterrey, Mexico, pages 35-36. Arlington, VA: NSF, January 1998.

[2] T. R. Jackson, N. M. Patrikalakis, E. M. Sachs, and M. J. Cima. Modeling and designing components with locally controlled composition. In D. L. Bourell et al, editor, Solid Freeform Fabrication Symposium, pages 259-266, Austin, Texas, August 10-12 1998. The University of Texas.

[3] J. R. Fessler, R. Merz, A. H. Nickel, and F. B. Prinz. Laser deposition of metals for shape deposition manufacturing. In D. L. Bourell et al., editor, Solid Freeform Fabrication Symposium, pages 117-124, Austin, Texas, August 12-14 1996. The University of Texas.

[4] K. J. Jakubenas, J. M. Sanchez, and H. L. Marcus. Multiple material solid free-form fabrication by selective area laser deposition. Materials and Design, 19(1/2):11-18, 1998.

[5] E. Sachs, J. Haggerty, M. Cima, and P. Williams. Three-dimensional printing techniques. U.S. Patent No. 5,204,055, April 201993.

[6] A. E. Giannakopoulos, S. Suresh, M. Finot, and M. Olsson. Elastoplastic analysis of thermal cycling: Layered materials with compositional gradients. Acta Metallurgica et Materialia, 43:1335-1354, April 1995.

[7] J. Yoo, K. Cho, W. S. Bae, M. J. Cima, and S. Suresh. Transformation-thoughened ceramic multilayers with compositional gradients. Journal of the American Ceramic Society, 81(1):2132, January 1998.

[8] I. Jackson, H. Xiao, M. Ashtiani, and L. Berben. Stereolithography model in presurgical planning of craniofacial surgery. In D. L. Bourell et al, editor, Solid Freeform Fabrication Symposium, pages 9-14, Austin, Texas, August 12-14 1996. The University of Texas.

[9] B. M. Wu, S. W. Borland, R. A. Giordano, L. G. Cima, E. M. Sachs, and M. J. Cima. Solid free-form fabrication of drug delivery devices. Journal of Controlled Release, 40(1/2):77-87, 1996.

[10] M. Mäntylä. An Introduction to Solid Modeling. Computer Science Press, Rockville, Maryland, 1988.

[11] J. R. Rossignac and A. G. Requicha. Offsetting operations in solid modelling. Computer Aided Geometric Design, 3(2):129-148, 1986.

[12] J. R. Rossignac and A. G. Requicha. Constructive non-regularized geometry. Computer Aided Design, 23(1):21-32, January/February 1991.

[13] M. Mäntylä. Advanced topics in solid modeling. In W. Purgathofer and J. Schönhut, editors, Advances in Computer Graphics V, chapter 3, pages 49-74. Springer-Verlag, Berlin, 1989. 
[14] IGES/PDES Organization, U.S. Product Data Association, Fairfax, VA. Digital Representation for Communication of Product Definition Data, US PRO/IPO-100, Initial Graphics Exchange Specification (IGES) 5.2, November 1993.

[15] American National Standards Institute. Product Data Exchange Using STEP (PDES) Part 42, Integrated generic resources: geometric and topological representation. Fairfax, VA, February 1995.

[16] E. L. Gürsöz, Y. Choi, and F. B. Prinz. Vertex-based representation of non-manifold boundaries. In M. J. Wozny, J. U. Turner, and K. Preiss, editors, Geometric Modeling for Product Engineering, pages 107-130, Holland, 1990. Elsevier Science Publishers.

[17] J. R. Rossignac and M. A. O'Connor. SGC: A dimension-independent model for point sets with internal structures and incomplete boundaries. In M. J. Wozny, J. U. Turner, and K. Preiss, editors, Geometric Modeling for Product Engineering, pages 145-180, Holland, 1990. Elsevier Science Publishers.

[18] V. Kumar and D. Dutta. An assessment of data formats for layered manufacturing. Advances in Engineering Software, 28(3):151-164, April 1995.

[19] V. Kumar and D. Dutta. An approach to modeling multi-material objects. In C. Hoffman and W. Bronsvort, editors, Fourth Symposium on Solid Modeling and Applications, Atlanta, Georgia, May 14-16, 1997, pages 336-353, New York, 1997. ACM SIGGRAPH.

[20] J. Pegna and A. Safi. CAD modeling of multi-modal structures for free-form fabrication. Presentation at Solid Freeform Fabrication Symposium, Austin, Texas, August 12-14, 1998.

[21] V. Kumar and D. Dutta. An approach to modeling and representation of heterogeneous objects. Journal of Mechanical Design, 120:659-667, December 1998.

[22] E. Brisson. Representing geometric structures in d dimensions: Topology and order. Discrete and Computational Geometry, 9:387-426, 1993.

[23] J. Hoschek and D. Lasser. Fundamentals of Computer Aided Geometric Design. A. K. Peters, Wellesley, MA, 1993. Translated by L. L. Schumaker.

[24] T. H. Cormen, C. E. Leiserson, and R. L. Rivest. Introduction to Algorithms. MIT Press, Cambridge, MA, 1990.

[25] H. Jee. Computer-Aided Design of Surface Macro-Textures for Three Dimensional Printing. PhD thesis, Massachusetts Institute of Technology, Cambridge, MA, April 1996.

[26] J. Neider, T. Davis, and M. Woo. OpenGL Programming Guide: the Official Guide to Learning OpenGL, Release 1. Addison-Wesley Publishing Company, Reading, Mass, 1993. 
Figure 1: (a) Conventional 3D Printing cycle. (b) A part with macro-textured surface fabricated through 3D Printing, demonstrating the geometric capability of the process.

Figure 2: 3D Printing cycle with multiple material delivery system. A print-head with multiple nozzles selectively deposits material into the print-bed.

Figure 3: (a) Model of tetrahedron. (b) Tetrahedron decomposed into cells. (c) Adjacency graph of cells.

Figure 4: A tetrahedral cell's (a) parametric domain, (b) control points in model space, (c) mapping of parametric domain into model space, (d) control compositions in material space, and (e) the resulting graded composition in model space.

Figure 5: 3 FGM cubes consisting of (a) degree $n_{m}=0$, (b) degree $n_{m}=1$, and (c) degree $n_{m}=3$ FGM cells. Each cube consists of 40 regions, 104 faces, 90 edges, and 27 vertices. 
Figure 6: Information flow from a traditional CAD system, through the FGM Modeler, and on to the 3D Printer.

Figure 7: (a) Running time of distance calculation versus number of query points using two methods: exhaustive search for nearest facet to the query point (dashed lines) and the bucketing technique (solid lines). The faceted model used for these computations contained $n_{b f}=15156$ boundary facets. (b) Model used for testing running time in minimum distance calculations. This model is an example part provided with SolidWorks ${ }^{T M}$.

Figure 8: Set of FGM Design Rules limiting volume fractions of material system and FGM model with violations of $D R_{2}$ indicated.

Figure 9: (a) Solid model of pulley. (b) Desired variation in composition as function of distance from axis of rotation. (c) View of FGM pulley. (d) Close up view of pulley decomposed into FGM cells. The volume fraction of carbide is proportional to the grey level in a cell

Figure 10: (a) Solid model of a pill. (b) Desired variation in composition as function of distance from the pill's boundary. (c) Exploded view of pill decomposed into FGM cells. (d) Slice view of FGM showing concentration of drug over internal tetrahedral cells. The volume fraction of drug is proportional to the grey level in a cell. 
Modeling and Designing Functionally Graded Material Components for Fabrication with Local Composition Control

T. R. Jackson, H. Liu, N. M. Patrikalakis, E. M. Sachs, and M. J. Cima

Figure 1

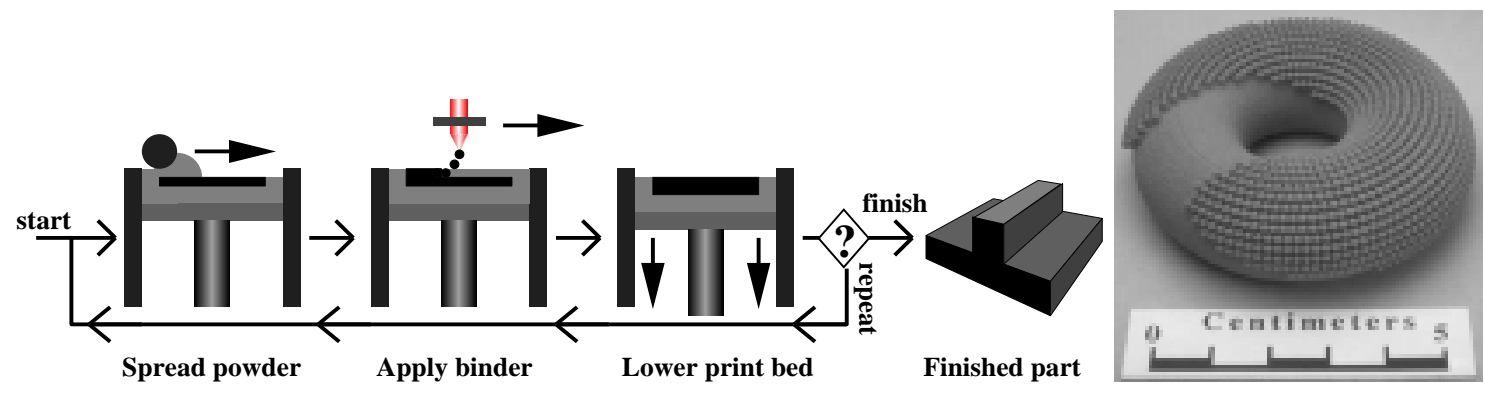


Modeling and Designing Functionally Graded Material Components for Fabrication with Local Composition Control

T. R. Jackson, H. Liu, N. M. Patrikalakis, E. M. Sachs, and M. J. Cima

Figure 2

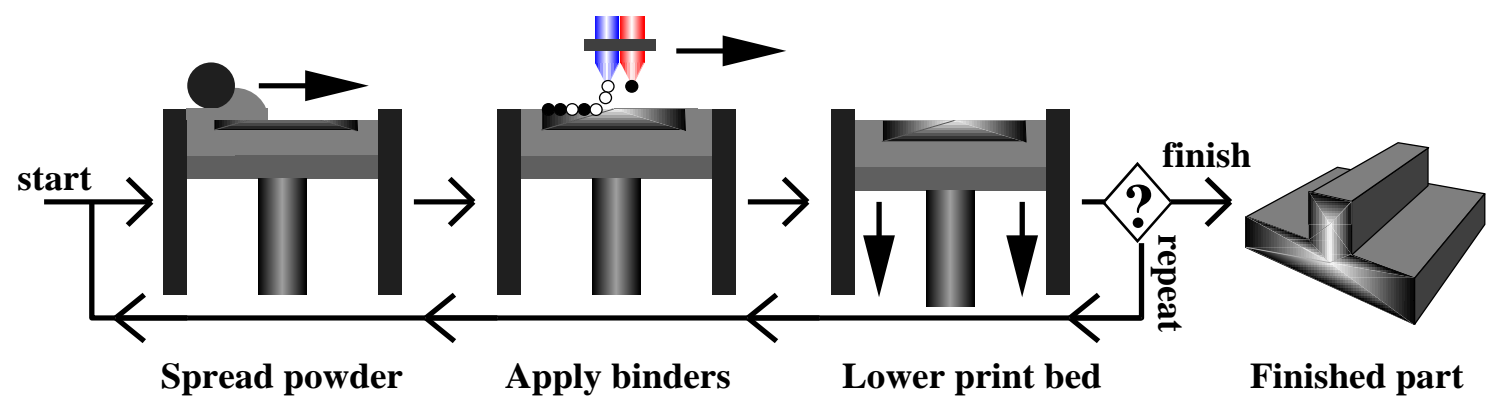


Modeling and Designing Functionally Graded Material Components for Fabrication with Local Composition Control

T. R. Jackson, H. Liu, N. M. Patrikalakis, E. M. Sachs, and M. J. Cima

Figure 3
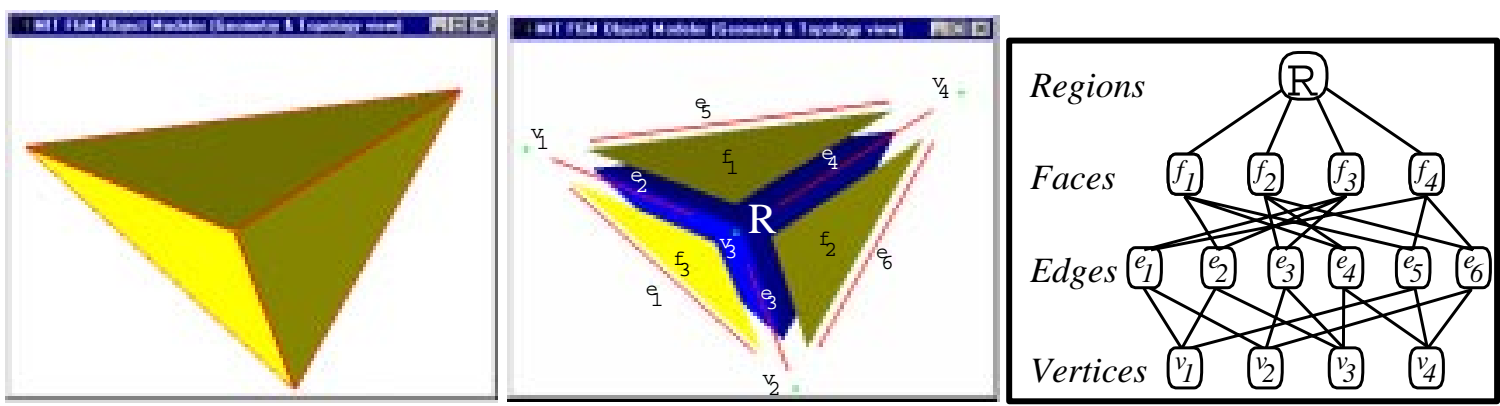
Modeling and Designing Functionally Graded Material Components for Fabrication with Local Composition Control

T. R. Jackson, H. Liu, N. M. Patrikalakis, E. M. Sachs, and M. J. Cima

Figure 4
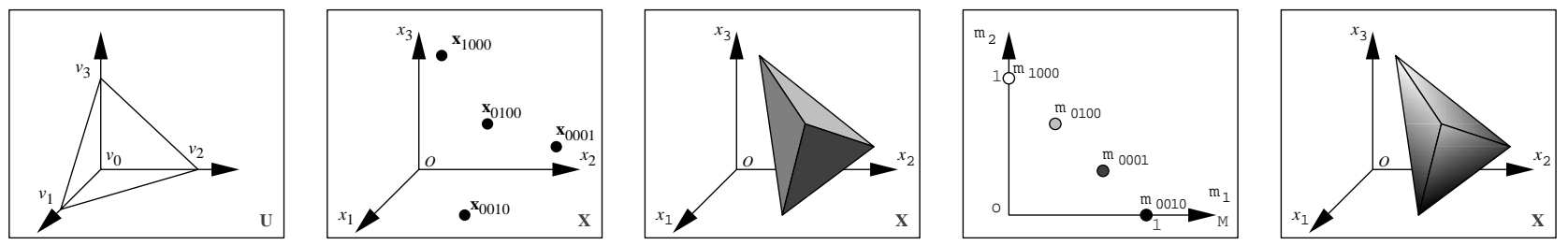
Modeling and Designing Functionally Graded Material Components for Fabrication with Local Composition Control

T. R. Jackson, H. Liu, N. M. Patrikalakis, E. M. Sachs, and M. J. Cima

Figure 5
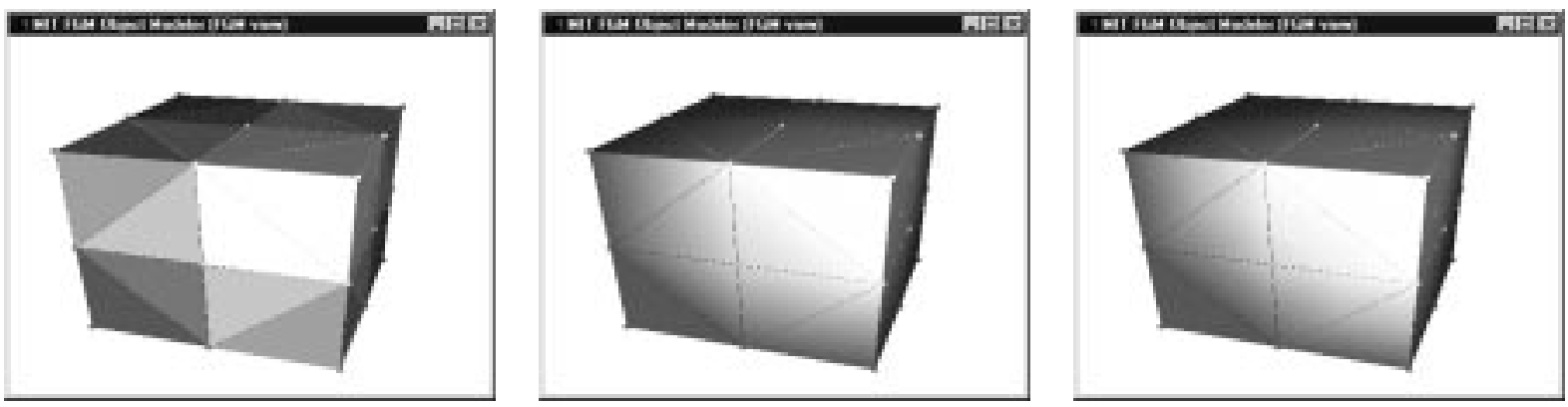
Modeling and Designing Functionally Graded Material Components for Fabrication with Local Composition Control

T. R. Jackson, H. Liu, N. M. Patrikalakis, E. M. Sachs, and M. J. Cima

Figure 6

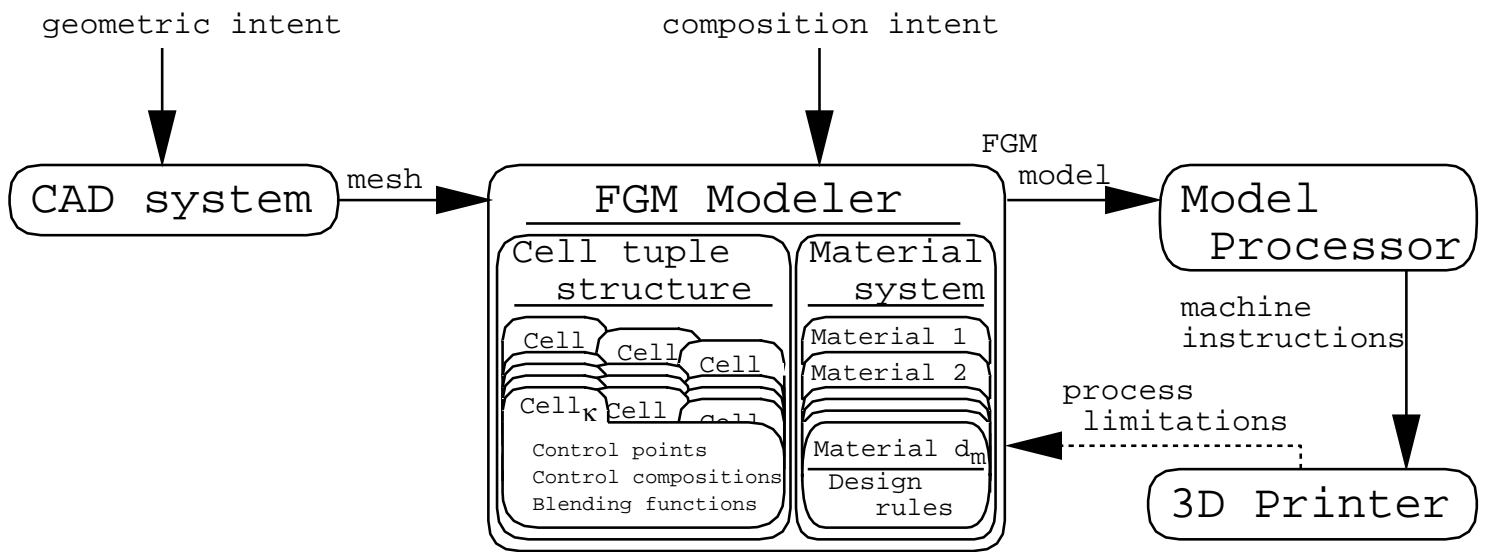


Modeling and Designing Functionally Graded Material Components for Fabrication with Local Composition Control

T. R. Jackson, H. Liu, N. M. Patrikalakis, E. M. Sachs, and M. J. Cima

Figure 7
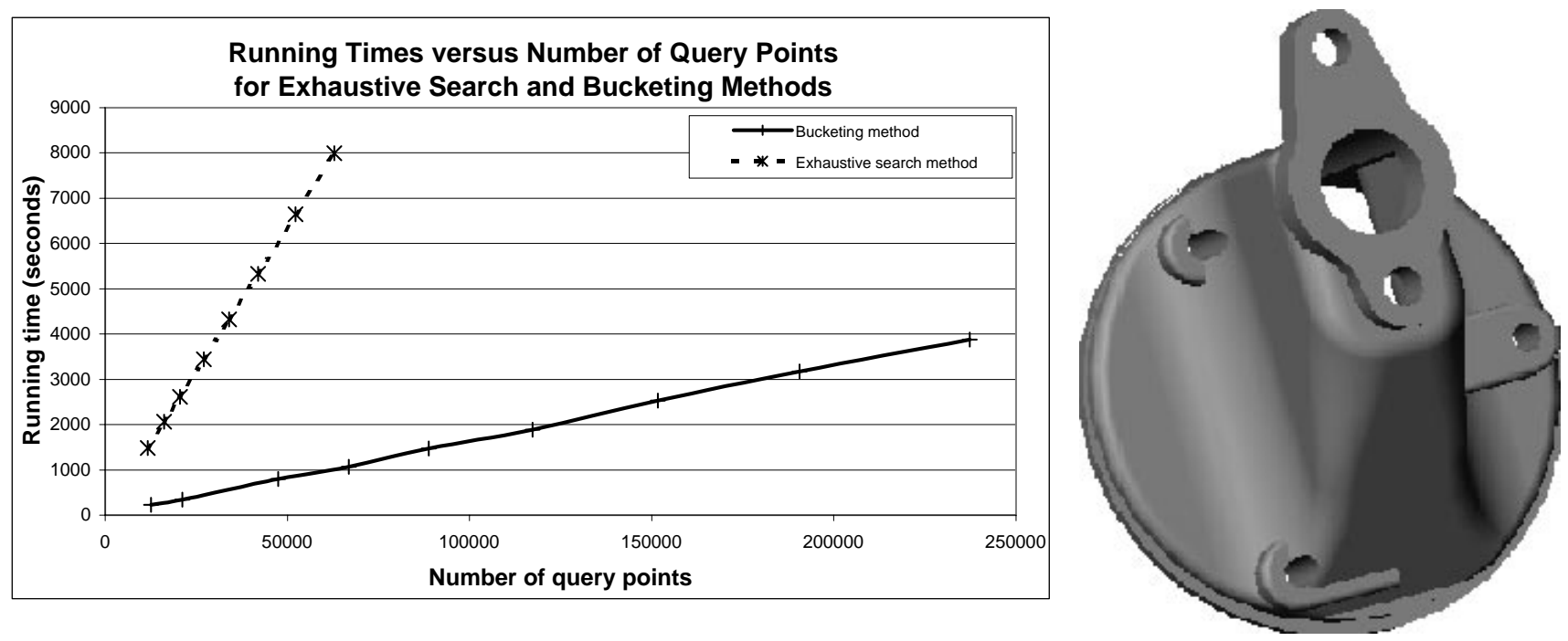
Modeling and Designing Functionally Graded Material Components for Fabrication with Local Composition Control

T. R. Jackson, H. Liu, N. M. Patrikalakis, E. M. Sachs, and M. J. Cima

Figure 8

\begin{tabular}{|c|c|c|c|}
\hline \multicolumn{4}{|l|}{ FGM: } \\
\hline \multirow{4}{*}{$\mathbf{m}(z)=$} & 0.65 & \multirow{4}{*}{$+\left|\frac{z}{z_{0}}\right|$} & 0.00 \\
\hline & -0.10 & & 0.00 \\
\hline & 0.00 & & 0.15 \\
\hline & 0.25 & & -0.15 \\
\hline \multicolumn{4}{|c|}{ Design Rules: } \\
\hline & Material & $\min$ & $\max$ \\
\hline$D R_{0}$ & $\overline{m_{0}}$ & 0.60 & 0.70 \\
\hline$D R_{1}$ & $m_{1}$ & 0.05 & 0.15 \\
\hline$\overline{D R_{2}}$ & $m_{2}$ & 0.00 & 0.10 \\
\hline
\end{tabular}

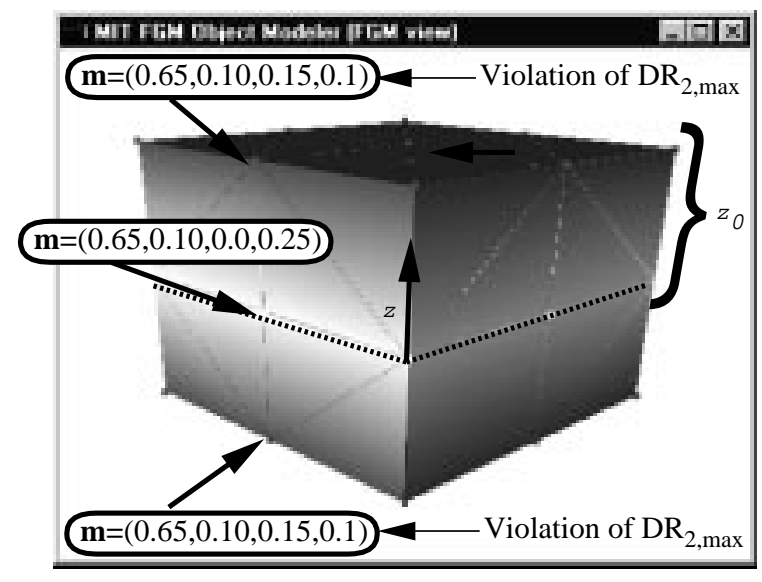


Modeling and Designing Functionally Graded Material Components for Fabrication with Local Composition Control

T. R. Jackson, H. Liu, N. M. Patrikalakis, E. M. Sachs, and M. J. Cima

Figure 9
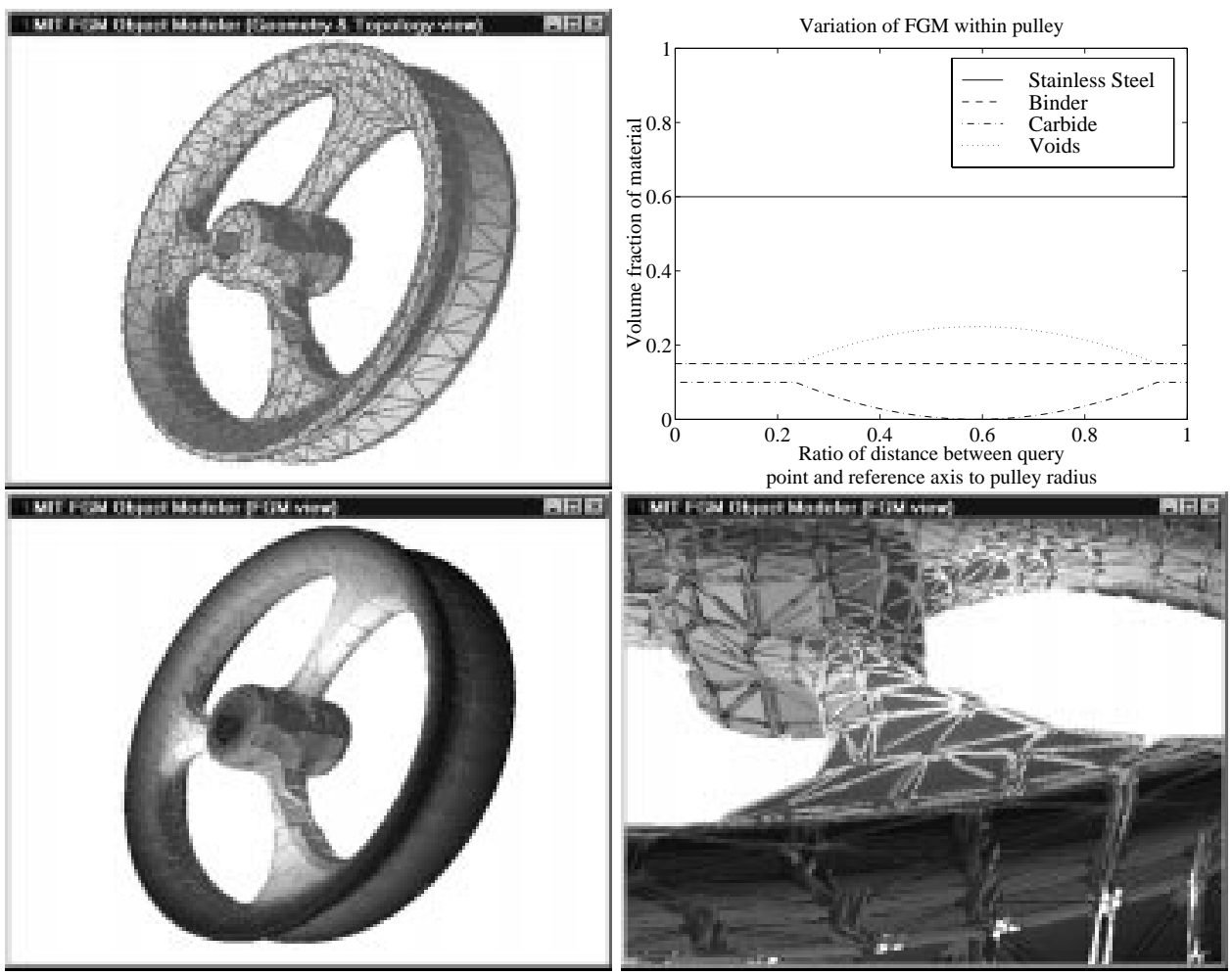
Modeling and Designing Functionally Graded Material Components for Fabrication with Local Composition Control

T. R. Jackson, H. Liu, N. M. Patrikalakis, E. M. Sachs, and M. J. Cima

Figure 10
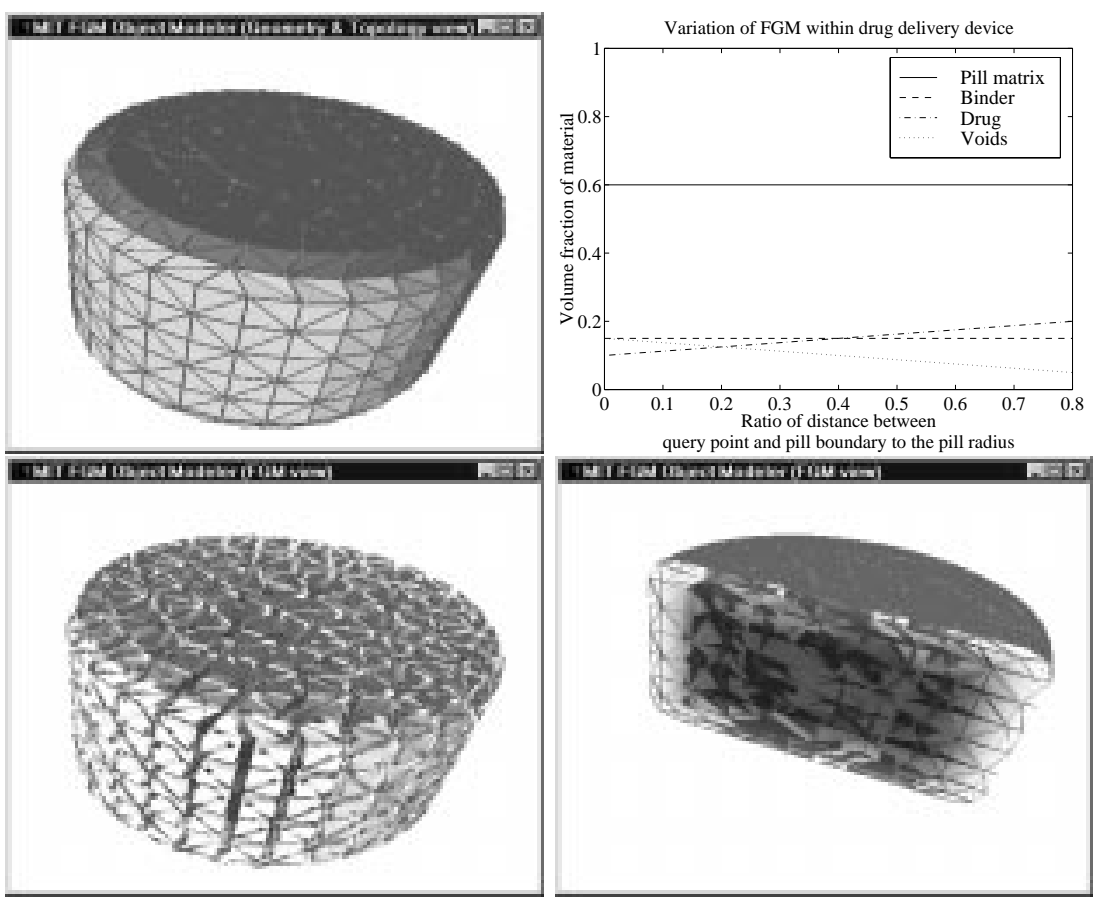